\title{
Efficacy of Xpert MTB/RIF Assay in the Diagnosis of Smear Negative HIV Seropositive Pulmonary Tuberculosis Patients
}

\author{
Reetika Bansal*, Shilpa Arora, Neerja Jindal, Renu Bansal and Vishal Sharma \\ Guru Gobind Singh Medical College \& Hospital, Faridkot (Punjab), India
}

\begin{abstract}
Background: Tuberculosis (TB) is the most common presenting illness and leading cause of death among people living with HIV(PLHIV). Diagnosis of TB among PLHIV is challenging as false negative results are common with direct microscopy (DM) and culture takes long time. WHO (2013) recommended Xpert MTB/RIF assay as an initial test for diagnosis of TB in PLHIV.

Methods: The present study was conducted on 142 HIV seropositive patients who were clinically suspected to have TB. The sputum specimens obtained from these patients were divided into two parts; one was processed by DM and the other was used for Xpert MTB/RIF assay.

Result: Of the 142 HIV seropositive patients, 90(63.3\%) were in the age group of 15-40 years and majority 91(64.08\%) were males. Out of 142 sputum specimens collected from these patients, only 5(3.52\%) were positive by DM and 31(21.83\%) by Xpert MTB/RIF assay. Statistically the difference between the two was significant ( $\mathrm{p}$ value $<0.005$ ). Xpert MTB/RIF assay showed rifampicin resistant in 2(6.45\%) Specimens. Of the 137 smear negative specimens, Xpert MTB/RIF assay gave positive result in 27 (19.70\%). Thus, the sensitivity, specificity, PPV, NPV of the Xpert MTB/RIF assay in comparison to DM was 80\% (95\%CI=37.6- 96.4), 80.29\% (95\%CI=72.8-86.1), 12.90\% (95\%CI $=4.21-30.76), 99.09 \%(95 \% \mathrm{CI}=94.3-99.9)$ respectively.

Conclusion: Xpert MTB/RIF assay was found to be a sensitive, specific and rapid method for diagnosis of TB and rifampicin resistance in smear negative PLHIV. It's availability as an initial diagnostic test in PLHIV could significantly reduce the delay in initiation of treatment and prevent the transmission of the disease.
\end{abstract}

Keywords: : Xpert MTB/RIF, Pulmonary Tuberculosis, Smear Negative, HIV,

\section{Introduction}

The estimated incidence of tuberculosis (TB) in India is approximately $28,00,000$ which accounts for about a quarter of the world's TB cases. ${ }^{[1]}$ Approximately 1.2 million new cases were notified in 2016 and of this $34 \%$ were HIV positive (WHO 2016). ${ }^{[2]}$ Diagnosis of TB among people living with HIV (PLHIV) is challenging as false negative results in these patients are common with direct microscopy (DM) which is the most common method of diagnosis of pulmonary TB. Culture for Mycobacterium tuberculosis (MTB) which is the current gold standard for diagnosis of tuberculosis is laborious and time consuming. It requires atleast 8 weeks before the results are obtained. In 2013, WHO recommended Xpert MTB/RIF assay as an initial test for the diagnosis of TB in adults and children suspected of having HIV associated TB. Xpert MTB/RIF assay is a cartridge based automated diagnostic test that can detect the presence of MTB as well as rifampicin resistance by amplification of the $81 \mathrm{bp}$ fragments of the MTB rpoB gene in less than 2 hours. It has been reported that the use of this technique had improved the quality of TB diagnosis among PLHIV in $36-75 \%$ of smear negative pulmonary TB patients. ${ }^{[3,4]}$ However, there are not many studies of similar nature from India which bears second highest number of HIV associated TB in world. ${ }^{[5]}$ Therefore, the present study was undertaken to determine the efficacy of Xpert MTB/RIF assay in diagnosing pulmonary TB in smear negative HIV seropositive patients attending the tertiary care hospital of Punjab (Northern India).

\section{Material and Methods}

A total of 1343 sputum Specimens collected from 1328 clinically suspected patients (between January 2016 and May 2017) were received in the department of Microbiology, Guru Gobind Singh Medical College, Faridkot (Punjab). Of these, 142 were from the patients who were found to be HIV positive by ELISA, Rapid and Simple (ERS) test according to National AIDS Control Organization (NACO) guidelines. ${ }^{[6]}$ The sputum specimens were processed by DM using Ziehl Neelsen (ZN) staining and by Xpert MTB/RIF assay. Stained smears were examined under oil immersion lens and grading was done on the basis of number of mycobacteria detected. For Xpert MTB/RIF assay sputum specimens collected in falcon tube were diluted with double the amount of sample reagent, shaken vigorously 10-20 times, incubated at room temperature for 10 minutes, shaken again and incubated 
further till these became perfectly fluid. The processed specimens $(2 \mathrm{ml})$ were pipetted to the cartridge and the cartridges were inserted in the machine to start the test. Results were available within 2 hours.

\section{Result}

The study of 142 HIV seropositive patients showed that most of them $90(63.3 \%)$ were in the age group of 15 40 years and majority 91(64.08\%) were males (Table 1). Out of 142 sputum specimens collected from these patients, $5(3.52 \%)$ were positive by DM and $31(21.83 \%)$ by Xpert MTB/RIF assay. Statistically the difference between the two was significant ( $p$ value $<0.005$ ). Xpert MTB/RIF assay showed rifampicin resistance in 2(6.45\%) specimens. Comparison of DM and Xpert MTB/RIF assay showed that out of $5(3.52 \%)$ sputum specimens positive by DM, $4(2.8 \%)$ were also positive by Xpert MTB/RIF assay. There was only one $(0.70 \%)$ specimen which was positive on DM but in which MTB was not detected by Xpert MTB/ RIF assay. On the other hand, of the 137 smear negative specimens, Xpert MTB/RIF assay gave positive result in $27(19.70 \%)$, while rest of the $110(77.46 \%)$ were negative for MTB. Thus the sensitivity, specificity, PPV, NPV of the Xpert MTB/RIF assay in comparison to DM was $80 \%$ (95\% CI=37.6- 96.4), 80.29\% (95\% CI $=(72.8-86.1)$, $12.90 \%(95 \% \mathrm{CI}=4.21-30.76), 99.09 \%(95 \% \mathrm{CI}=94.3-99.9)$ respectively. The two samples which showed rifampicin resistance were also smear negative. (Table 2)

\section{Discussion}

Tuberculosis remains a major public health problem in the developing countries. The situation has worsened further with the emergence of HIV/AIDS and multidrug resistant tuberculosis (MDRTB). Infection with HIV has greatly increased the risk of developing tuberculosis and accelerating its progress to AIDS. Therefore, effective diagnosis of TB in HIV positive patient is the priority to increase case detection \& to improve treatment outcome.

In the present study, 142 HIV positive patients suspected of having TB were studied. Maximum (63.3\%) of them were in the age group of 15-40 years. This is almost similar to the studies conducted in Maharashtra (India) and Namibia. ${ }^{[7,8]}$ The predominance of this age group could be due to the fact that this age group is sexually active and may be encountering sexual partners with HIV infection. Majority (64.08\%) of these patients was males and male to female ratio was 1.7:1(Table 1). Bhadke et al observed this ratio as 2.73:1. ${ }^{[7]}$ The predominance of males in HIV/ TB co-infection has its impact on the loss of economic productivity of the society.

The traditional diagnosis of HIV/TB is complex, expensive and slow as the coinfected (HIV/TB) patients have disease which is paucibacillary in nature and present with atypical radiographic manifestations. In the present study the ageold DM technique detected TB in only 5 of $142(3.52 \%)$ HIV positive patients. However, when Xpert MTB/RIF assay was used it increased the detection rate to $21.83 \%$ and the difference was statistically significant (Table 2). The results are consistent with other studies and literature which state that DM has limited value in the diagnosis of TB. ${ }^{[4,8]}$ On the other hand, there was one $(0.70 \%)$ case of

Table 1: Age \& Sex wise distribution of HIV seropositive patients $(n=142)$.

\begin{tabular}{|c|c|c|c|}
\hline Age Group & Males & Females & Total \\
\hline $0-14$ & 3 & 0 & $3(2.1 \%)$ \\
\hline $15-40$ & 52 & 38 & $90(63.3 \%)$ \\
\hline $41-60$ & 35 & 13 & $48(33.8 \%)$ \\
\hline$>60$ & 1 & 0 & $01(0.7 \%)$ \\
\hline Total & $\mathbf{9 1 ( 6 4 . 0 8 \% )}$ & $\mathbf{5 1 ( 3 5 . 9 2 \% )}$ & $\mathbf{1 4 2 ( 1 0 0 \% )}$ \\
\hline
\end{tabular}

Table 2. Comparison of Direct Microscopy and Xpert MTB/RIF assay

\begin{tabular}{|c|c|c|c|c|}
\hline & & \multicolumn{3}{|c|}{ Direct Microscopy } \\
\hline & & Positive & Negative & Total \\
\hline \multirow[t]{3}{*}{ Xpert MTB/RIF assay } & Positive & 4 & 27 & $31(22.08 \%)^{b}$ \\
\hline & Negative & 1 & 110 & $111(78.16 \%)$ \\
\hline & Total & $5(3.52 \%)^{a}$ & $137(96.47 \%)$ & $142(100 \%)$ \\
\hline
\end{tabular}

$p$ value between $a$ and $b<0.005$ (significant)

- $\quad$ Sensitivity $=80 \%(95 \% C I=37.6-96.4)$

- $\quad$ Specificity $=80.29 \%(95 \% C I=72.8-86.1)$

- $\quad$ Positive predictive value $=12.9 \%(95 \% C I=4.21-30.76)$

- $\quad$ Negative predictive value $=99.09 \%(95 \% \mathrm{CI}=94.3-99.9)$ 
TB in the present study which was detected by DM but Xpert MTB/RIF assay showed it as MTB not detected. This could be because Xpert MTB/RIF assay targets only MTB while DM is positive with all acid fast bacilli including non tubercular mycobacteria.

In the present study, $27(19.70 \%)$ smear negative HIV positive patients of pulmonary TB(SN-PTB) were detected by Xpert MTB/RIF assay. (Table2) These findings are in agreement with those of other studies and give an extra egde to the efficacy of Xpert MTB/RIF assay to diagnose MTB in smear negative cases. ${ }^{[7,8]}$ False negative results in DM in such a large number causes delay in the diagnosis and initiation of prompt antitubercular treatment in these HIV positive patients who are already at increased risk of morbidity and mortality because of co-infection.

Taking Direct Microscopy as gold standard (as culture was not routinely performed) sensitivity and specificity of Xpert MTB/RIF assay was found to be $80 \%$ and $80.29 \%$ respectively (Table2) which is comparable to that reported by WHO (sensitivity and specificity of $80 \%$ each). ${ }^{[9]} \mathrm{A}$ study conducted by Ioannidis et al reported slightly higher values of sensitivity $(90.6 \%)$ and specificity $(94.3 \%)$ for Xpert MTB/RIF assay ${ }^{[10]}$ Kakoma et al found the sensitivity to be high $(98 \%)$ while the specificity was low $(54 \%){ }^{[8]}$

Rifampicin resistance was observed in 2(6.45\%) PLHIV in comparison to the studies of Nguyen et al and Bansal et $a l$ who reported rifampicin resistance in $3.7 \%$ and $0.99 \%$ respectively. ${ }^{[11,12]}$ Since Xpert MTB/RIF assay gives result within 2 hours, treatment initiation of MDR-TB in people living with HIV could be significantly shortened thus, reducing premature death and ongoing transmission of tuberculosis.

\section{Conclusion}

Screening of pulmonary specimens of all HIV positive patients with Xpert MTB/RIF assay has enormous scope in terms of finding active cases of new tuberculosis patients and furthermore those with drug resistant tuberculosis. For patients who are smear negative, utilizing Xpert MTB/ RIF assay may provide a rapid diagnosis that might have otherwise missed on DM. Although the drug resistance may be similar in seropositive and seronegative patients, still timely detection of drug resistance in HIV helps in reducing morbidity in HIV/TB co-infection.

\section{Reference}

1. Global tuberculosis report 2017. Geneva: World Health Organization; 2017. Licence: CC BY-NCSA 3.0 IGO.

2. Global tuberculosis report 2016. Available at http://apps. who.int /medicinedocs / documents/s23098en/s23098en. pdf. accessed on 15 March 2018.

3. Xpert MTB/RIF for people living with HIV. Available at:http://www.who. int/tb/challenges/hiv/Xpert_TBHIV_ Information_Note_final.pdf.Accessed 21 April 2017.

4. Dewan R, Anuradha S, Khanna A, Garg S, Singla S, Ish P, et al. Role of cartridge-based nucleic acid amplification test (CBNAAT) for early diagnosis of pulmonary tuberculosis in HIV. JIACM. 2015; 16(2):114-7.

5. TB India 2017, Annual status report, Central TB division, chapter $2 \mathrm{https}: / /$ tbcindia.gov.in/writereaddata/TB\%20 India\%202017.pdf

6. National Guidelines for HIV Testing. Available at:http:// www.naco.gov .in/sites/default/files/National_Guidelines for_HIV_Testing_21Apr2016.pdf. Accessed 22 March 2017.

7. Bhadke BB, Rathod RK, Mulay SD, Deshmukh DG, Mahajan PD. Efficacy of Gene Xpert in diagnosing MTB/ RIF resistance in HIV seropositive and seronegative patients: a study in comparison. International Journal of Medical Microbiology and Tropical Diseases . 2017; 3(2):50-6.

8. Kokoma LN, Mukesi M, Moyo SR. Effectiveness of Gene Xpert technology in the diagnosis of smear-negative pulmonary mycobacterium tuberculosis in HIV positive patients in Namibia. Open J Med Microbiol. 2016; 6(3):133-41.

9. WHO (2011) Automated real-time nucleic acid amplification technology for rapid and simultaneous detection of and rifampicin resistance: Xpert MTB/RIF System.

10. Ioannidis P, Papaventsis D, Karabela S, Nikolaou S, Panagi M, Raftopoulou E, et al. Cepheid Gene Xpert MTB/RIF assay for Mycobacterium detection and rifampin resistance identification in patients with substantial clinical indications of and smear-negative microscopy results. J Clin Microbiol. 2017; 49:3068-70.

11. Ngyuyen T, Dorothee H, Do Dang T, Tran T, Nguyen TH, Ho Dang $T$ et al. Evaluation of Gene Xpert MTB/RIF for Diagnosis of Tuberculosis Meningitis. J Clin Microbiol. 2014; 52(1):226-33.

12. Bansal D, Avashia S, Karothiya M. A study of Gene Xpert in screening of sputum in HIV positive patients presenting to tertiary care centre. National J Community Med. 2016; 7(8):657-60.

*Corresponding author:

Dr. Reetika Bansal, Department of Microbiology, 2nd floor, Guru Gobind Singh Medical College, Faridkot.

Phone: +91 9041222242

Email: reetika.bansal11@gmail.com

Financial or other Competing Interests: None. 